average civil surgeon in India. Slight variations in the technique of this operation may produce large changes in the result, and though this is an advantage in the hands of the trained operator, suiting various grades and types of glaucoma, it is disconcerting to the beginner.

The method has a very obvious advantage over trephining, as will be readily understood from the growing recognition of the importance of spreading sub-conjunctival drainage over a wide area. And, as I have realised lately, it is particularly suited for use at the site of a previous wide iridectomy. The very narrow knife can be freely manipulated in the periphery of the anterior chamber without the slightest risk of injury to the lens. But, particularly, perhaps, where there may be some conjunctival adhesion from former operation, I think the covering conjunctival flap should be more freed above than in my old description.* Early elastic retraction of the flap may then be expected to cause slight bulging from accumulation of aqueous beneath it, thus producing a very desirable slight forward displacement of the sclero-corneal wedge.

Of the $16(19-3)$ results included in my lists, 5 were in the recurrent and 11 in the non-recurrent list. The mean period since operation was 11 and 12 years respectively in the two groups. Three of the 5 recurrences were so recent and so intermittent as to have had little or no permanent effect on vision.

The feature which impressed one most in the whole series of these particular iris-free cicatrices was their very satisfactory appearance. There were no dangerous-looking scars. There were perfect examples of filtering lines still outlining the wedges. And in cases in which, a few months after operation, disappearance of ill-placed detached wedges had led to ugly and threatening sclerocorneal gaps, with hypotony, there were now the narrowest of slits - under practically normal conjunctiva, only doubtfully distinguishable from true filtration. Possibly the change may have occurred almost entirely during the first two year's after operation.

I have known of two late infections after this operation, but they were both mild and transient.

\title{
ANNOTATIONS
}

\section{Motor Headlights}

The important question of the best form of headlight for motor vehicles formed the subject of an interesting debate at a recent

*The Ophthalmoscope, Vol. V. (1907), p. 298. 
meeting of the Illuminating Engineering Society. Major Garrard read a paper on the various optical measures that have been taken to reconcile the requirements of the driver who needs a powerful beam to distinguish objects ahead, and those of pedestrians or drivers of other vehicles who are apt to be dazzled by the brilliancy of the beam. The only practical solution at present available is one which limits the vertical height of the beam and this is the aim of the regulations at present in force in America, in which country, owing to the greater number of motor vehicles in use, the question is even more urgent than here. If the vertical height was all that mattered the problem would present no great complexity, but, as Major Garrard went on to point out, the satisfactory beam must be a composite one and must satisfy the three following conditions: " 1 . A fairly wide but relatively dim beam with a well-defined upper limit 3 or 4 feet from the ground so as to illuminate the hedges and ditches and the road in front of the car. This beam should preferably be of varying intensity so as to direct more light upon the more remote objects in, say, the middle distance. 2. A central but shallow penetrating beam of much greater intensity with a well-defined upper limit 3 or 4 feet from the ground for distance observation. This might extend about four degrees on each side of the axis, although it is difficult to suggest exact limits as the best results are probably obtained when the bright beam is shaded off fairly steeply into the wide beam. 3. A diffused or subdued beam having a very wide angle and corresponding to the light obtained direct from the filament of an ordinary electric headlamp without reflection." He did not attach much importance to 3 , but considered that it might have a certain psychological effect, an effect that might also follow the use of a comparatively bright beam inclined upwards at such an angle that it is above the eyes of other road users. After discussing the various methods in use he concluded that a sufficiently definite line of demarcation between light and darkness is only likely to be obtained commercially by the use of at least one spherical, focussing or projecting lens or objective, and under this heading is included the Mangin mirror; which may be regarded as a combination of a reflector and a focusing lens. In the subsequent discussion some attention was drawn to the R.A.C. standard anti-dazzle disc for testing motor lamps, and specimens of lamps were shown. Possibly the time is not yet ripe for legislation on the subject, but as the number and speed of motor cars using the roads at night increases it would be of some value if an authoritative body such as the R.A.C. would take up the question of testing lamps and of issuing certificates with such lamps as proved satisfactory. 